\title{
An Algorithm to Optimize Pain Detection and Management in Older Patients in Routine Practice
}

Sylvie Bonin-Guillaume ${ }^{1,2,{ }^{*}}$, Patrice Rat ${ }^{1}$

1. Internal and Geriatric Medicine Department, University Hospital of Marseille, Hôpital de SainteMarguerite, 270, bd Sainte-Marguerite, 13009 Marseille Cedex, France; E-Mails:

sylvie.bonin@ap-hm.fr; patrice-rat@orange.fr

2. Institut Neurosciences des Systèmes, UMR-INSERM 1106, Aix-Marseille University, 27, bd JeanMoulin, 13005 Marseille Cedex, France

* Correspondence: Sylvie Bonin-Guillaume; E-Mail: sylvie.bonin@ap-hm.fr

Academic Editor: James S. Powers

Special Issue: Pain and Pain Management in the Elderly

\section{OBM Geriatrics}

2020, volume 4, issue 4

doi:10.21926/obm.geriatr.2004141
Received: July 09, 2020

Accepted: November 22, 2020

Published: December 07, 2020

\begin{abstract}
Acute or persistent pain is a common occurrence and is often undertreated in older patients, especially those with an inability to communicate verbally (ICV). Regular comprehensive pain assessment, including self-rating and/or behavior scales, is critical but difficult to implement in routine clinical practice. The choice of the most appropriate scale for each patient is not easy, even for trained and skilled medical personnel. Indeed, the use of scales for short painbehavior exposes to pain under-detection due to false-negative results, whereas extensive behavior scales are time-consuming, require pre-rating knowledge of the patient, and can also yield false-positive scores because of an overlap between pain-associated behaviors and those of other non-painful conditions (i.e., dementia, delirium, or depression). We describe the process used to devise an at-a-glance algorithm targeting medical personnel. This algorithm combines short and long pain scales specifically validated for geriatric populations with ICV or without. This algorithm has been tested by health workers in several settings. The final version of this algorithm reliably detected nociceptive acute or chronic pain in older patients and can
\end{abstract}

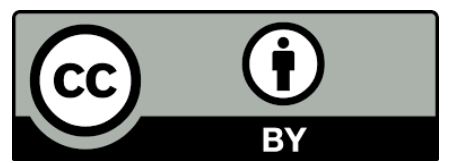

(c) 2020 by the author. This is an open access article distributed under the conditions of the Creative Commons by Attribution License, which permits unrestricted use, distribution, and reproduction in any medium or format, provided the original work is correctly cited. 
easily be applied to older patients in routine practice in any setting. This algorithm ensured a rapid and easy-to-use comprehensive assessment of pain in older patients to determine the need for analgesic administration.

\section{Keywords}

Pain assessment; algorithm; older patients; cognitive impairment; pain behavior scales

\section{Introduction}

Acute or persistent pain is common in older persons but remains under-recognized and undertreated, especially in those with an inability to communicate verbally (ICV). Yet, particularly for geriatric populations, good pain management must target all pain conditions, evaluate pain with appropriate tools to deliver effective analgesic treatment, then monitor its management with reassessment. Pain management is particularly important in older individuals with ICV, a situation that includes not only patients with dementia but also those with aphasia, delirium, coma, and/or poor understanding of the local language.

Self-assessment should be done first as the patient is his/her own pain expert. The "gold standard" Visual Analogic Scale for assessing pain in older individuals has major limitations, especially poor understanding of instructions. The Verbal Descriptor Scale is more reliable, the most preferred, and the easiest for older subjects with cognitive impairment [1].

In 2002, the American Geriatrics Society recommended six behavior domains (i.e., facial expressions, verbalizations/vocalizations, body movements, changes in interpersonal interactions, changes in activity patterns/routines, and mental status changes) to evaluate pain in seniors with dementia [2].

For the past two decades, many authors have reported on pain-behavior tools. Among 30 different scales were developed and tested, and only a few defined a cut-off score to determine the need for an analgesic. A step-by-step validation of measuring tools is a process requiring specific procedures to evaluate psychometric properties, but, unfortunately, most lacked validity because of major methodological limitations [3]. Still, no behavior pain-scale gold standard exists.

Despite the numerous pain-assessment scales available, the screening of pain by medical staff is still underperformed in routine practice. That statement is supported by a recent French multicenter survey conducted in 53 geriatric settings (acute, long-term and rehabilitation care, and nursing homes) that recruited 854 older patients receiving treatment for pain: $11 \%$ were not assessed for pain, and only $17.5 \%$ were evaluated with a behavior scale, despite $68 \%$ having ICV [4]. Those findings warrant two comments: pain assessment and reassessment still need to be strongly encouraged in geriatric settings, despite clinical and/or organizational barriers (i.e., timeconsuming), and the choice of the proper scale for each patient is crucial to assuring the continuing implementation and usage of pain-assessment tools [5].

In older individuals with ICV, the choice between short and long pain-behavior tests can be problematic. (1) Brief rating scales are widely applied in clinical practice in every setting as they are easy to use and rapid, but they can yield false-negative scores, especially in long-term care settings. (2) Extensive pain-behavior scales evaluate chronic pain conditions, which increases the workload 
of medical personnel [6]. Such patients must be tested, mainly in long-term care and nursing homes. However, they are time-consuming, require pre-rating knowledge of the patient, and can also yield false-positive scores, reflecting an overlap between pain-associated behaviors and those of other non-painful conditions (i.e., dementia, delirium, or depression [7]).

Therefore, a rapid, high-performance algorithm is needed to help healthcare workers choose the right tool for the right patient, avoiding time lost on assessments.

Some recent studies have described decision trees but for specific and various domains of pain identification and management, e.g., pain predictors for arthritis [8], postoperative pain management [9], or prediction of analgesic pain control [10]. Based on a modified Delphi method, a panel of experts advanced some recommendations for geriatric patients with chronic low back pain and suspected of having neurocognitive disorders (i.e., dementia) [11]. However, those studies required either the examiner's expertise or time to assess all the contributors or only deal with pain related to a specific pathology.

To the best of our knowledge, no simple and ubiquitous algorithm has been reported focusing on routine practice in different settings for better pain-assessment tools to be incorporated into healthcare workers' panoply of tools for regular assessment of pain in seniors. The aim of this short communication was to describe a new approach to devise an at-a-glance algorithm to better evaluate acute and/or chronic nociceptive pain and initiate analgesic treatment for older patients with or without ICV.

\section{Methods}

During the decade of 2010s, a brief and easy-to-use short behavior scale was specifically developed and validated to assess acute and chronic pain in geriatric patients, not only with cognitive impairment but also with other ICV situations. The 5-item Algoplus scale meets the validation standards for good psychometric properties. A cut-off score of $2 / 5$ recommends analgesic administration, with good sensitivity-to-change under treatment [12], and reliably assesses pain in patients with depression and/or dementia $[13,14]$. This scale was tested in emergency rooms, acute-care and long-term care units (Table 1) and showed good inter-rater validity (Table 2), even without any specific training (scoring time: $<1 \mathrm{~min}$ ). Finally, the Algoplus scale was recently translated and validated in five languages [15]. Thus, this scale is a good candidate to assess acute and chronic pain in patients with ICV or without.

Table 1 Sensitivity and specificity of an Algoplus cut-off score of 2 or 3 according to setting and patient group [from 16].

\begin{tabular}{|c|c|c|c|c|}
\hline \multirow{2}{*}{$\begin{array}{l}\text { Setting } \\
\text { Patients }\end{array}$} & \multicolumn{2}{|c|}{ Cut-Off Score $=2$} & \multicolumn{2}{|c|}{ Cut-Off Score $=3$} \\
\hline & Sensitivity & Specificity & Sensitivity & Specificity \\
\hline \multicolumn{5}{|c|}{ Emergency rooms ( $n=94)$} \\
\hline All patients & 88 & 71 & 67 & 93 \\
\hline ICV patients $(n=41)$ & 100 & 53 & 88 & 87 \\
\hline \multicolumn{5}{|c|}{ Geriatric acute-care units ( $n=179)$} \\
\hline All patients & 93 & 89 & 75 & 98 \\
\hline ICV patients $(n=108)$ & 96 & 86 & 76 & 96 \\
\hline Long-term-care settin & & & & \\
\hline
\end{tabular}




\begin{tabular}{lllll} 
All patients & 69 & 86 & 61 & 100 \\
ICV patients $(n=66)$ & 69 & 82 & 61 & 100 \\
\hline
\end{tabular}

ICV, inability to communicate verbally. Areas under the curve for all and ICV patients, respectively: 0.892, 0.935, in emergency rooms; 0.944, 0.959, in acute-care units; and 0.836, 0.827 , in long-term care facilities) [from 12].

Table 2 Interrater reliability of 533 paired evaluations according to the different medical personnel*.

\begin{tabular}{llll}
\hline Partner & Nurses & Nurse's aides & Physicians \\
\hline Nurses & $0.911^{\dagger}$ & 0.85 & 0.88 \\
Nurses 'aides & 0.85 & 0.93 & - \\
Physicians & 0.88 & - & 0.85 \\
\hline
\end{tabular}

*Nurses-nurse's aides: 21\%; nurses-physicians: 30\%; nurses-nurses: $32 \%$; physiciansphysicians: 10\%; nurse's aides-nurse's aides: 7\%) [from 17]. † Kappa coefficient correlation.

However, because of the lower sensitivity in long-term care (Table 1), a risk of undetected pain exists if Algoplus is used alone [18]. Hence, a combination of short and long scales in that setting was discussed, with the aim of reinforcing efficient, high-performing pain assessment [18]. That situation highlights the need for an algorithm to improve pain-evaluation quality for older adults in daily practice and lower the number of individuals in pain not receiving treatment, especially those with ICV.

This study was based on clinical studies that met ethical requirements for patient information and agreement to participate.

\section{Results}

French geriatricians and pain experts elaborated the first version of an algorithm for a comprehensive pain assessment in older individuals with ICV. Every older participant was asked to self-report pain, which had to be confirmed with a brief behavior scale before starting pain management. When medical personnel (nurses, physicians, nurses' aides) found self-reporting to be inaccurate, a brief behavior scale (i.e., Algoplus) was used systematically. When the Algoplus score was $\geq 2$, pain management was recommended (analgesic prescription and reevaluation). When the Algoplus score was <2, pain detection was combined with an extensive behavior tool (i.e., Doloplus, the Behavioral Scale for Elderly Persons (ECPA), or Pain Assessment Checklist for Seniors with Limited Ability to Communicate (PACSLAC) scales) [19].

When that first algorithm version was applied in nursing homes, pain-rating-scale implementation as a routine practice was better (particularly compared to the long behavior scales). Nevertheless, the need to use longer scales for an Algoplus score < 2 (i.e., 1 or 0 ) was still too frequent, ranging from $45 \%$ [13] to $60 \%$ [18] and, therefore, unsuitable because of the long time required to assess those patients.

To improve the algorithm's pertinence by optimizing the time allocated for pain assessment and limiting the risk of undetected patients in pain, the use of a long pain-behavior scale was limited to 
when the Algoplus score was 1 . Thus, for an Algoplus score of $1 / 5$-and only in this case-to avoid the risk of a false-negative result, pain status had to be confirmed with an extensive behavior scale (Doloplus-10 element or PACSLAC-multielement) before considering analgesic treatment. As for several other scales, an Algoplus score of 0 means the risk of undetected pain is extremely low $(<5 \%$ compared to a positive Doloplus score, according to real-life testing in long-term care, unpublished data).

This final version of the algorithm (Figure 1) specifically targets healthcare workers to obtain systematic pain evaluations in most-if not all-situations, regardless of setting.

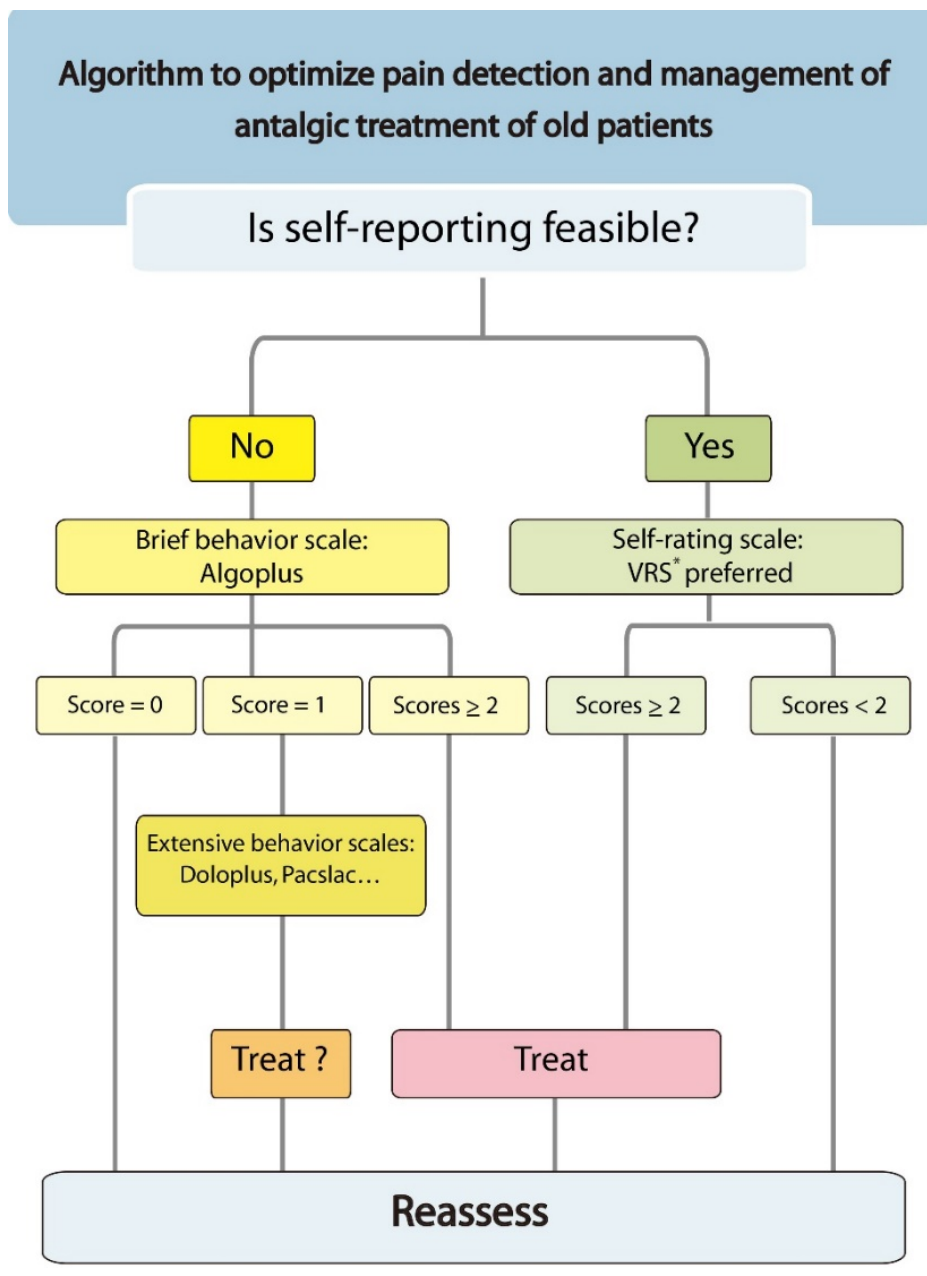

Figure 1 Algoplus algorithm for pain assessment in older subjects without or with inability to communicate verbally. ${ }^{*}$-point Verbal Rating Score (VRS).

Briefly, the first step is to assess pain with the 5-point Verbal Rating Scale (a kind of Verbal Descriptor Scale) and confirm it, if possible, by a second assessment. If the pertinence of the results is uncertain, then go to step 2.

The second step is to choose a pain-behavior scale. First, apply a brief but reliable tool with a cutoff score that identifies pain: (1) an Algoplus score $\geq 2$ ascertains the presence of pain that reliably requires analgesic treatment [12]; (2) an Algoplus score of 1 requires a complementary assessment with an extensive pain-behavior scale; (3) an Algoplus score of 0 ascertains no pain. Regular monitoring must be scheduled for every situation. 


\section{Discussion}

To the best of our knowledge, the Algoplus algorithm is a reliable acute and chronic paindetection tool for detecting pain in older patients with ICV or without. It was constructed using three different pain-rating scales with good psychometric properties and a solid validation process based on several multicenter national studies. The contribution of threshold scores at each step also assures better-adapted analgesic prescription to relieve pain.

Systematic evaluations are important for nurses' mastery of pain-rating scales [5]. The choice to limit the use of long behavior scales (i.e., to reduce risk of undetected pain in old patients) only for an Algoplus score of 1 (uncertain pain status) is a real-life approach to limit the time required to assess potential pain, with a low risk of pain going undetected in older patients. We think that this algorithm can be easily and quickly applied in routine practice to improve systematic assessments and reassessments on a regular basis, which are often lacking, particularly in a geriatric setting, going counter to international and national recommendations [20,21].

Other brief (self-reporting or and hetero-evaluation) scales commonly used in geriatric settings could be implemented in this algorithm, provided they have undergone complete validity processing and accuracy testing to decide analgesic treatment in different elderly populations and for acute and chronic pain.

However, this algorithm cannot be implemented in intensive care units, where populations are different, and specific pain-assessment tools are needed because pain behaviors in that setting might be different $[22,23]$.

The Algoplus algorithm is not limited to patients with dementia but considers all ICV patients, unlike most other scales. Even though medical personnel are trained and possess developed skillsets, they face difficulty identifying pain in ICV patients and may be unsure of the relevance of self-rating results [5]. To the best of our knowledge, no consensual definition of patients with ICV exists. We retained different clinical situations responsible for ICV that are commonly found in geriatric settings (and emergency rooms) [12], which could be another limitation. However, specific studies on this topic are still needed to better recognize and treat these patients.

At present, with the step-by-step development process described, this algorithm should help healthcare workers use the right tool (pain-behavior or self-assessment) for the right patient (with ICV or without) at the right time (importance of assessment and reassessment) and accurately adapt the need for analgesic treatment (appropriate or inappropriate analgesia). It is crucial to limit the risk of unrelieved pain and its consequences in those patients (depression, delirium, functional disability, etc.) [24].

\section{Conclusions}

This at-a-glance algorithm assured comprehensive and regular pain (re)assessment(s) in older patients with ICV or without in daily practice to determine the need to prescribe analgesic therapy. The next step would be to conduct implementation studies using this algorithm in different settings.

\section{Acknowledgments}

Authors acknowledge all the contributors to the Algoplus validation process and algorithm testing, more specifically, Marie Suzanne Leglise; Elisabeth Jouve for her methodological expertise 
on validation studies; and Janet Jacobson for editorial assistance.

\section{Author contribution}

Sylvie Bonin-Guillaume and Patrice Rat contributed equally to the planning and authorship of this manuscript.

\section{Funding source}

This specific research did not receive any specific grant from funding agencies in the public, commercial or not-for-profit sectors.

\section{Competing interests}

The authors declare that no competing interest exists.

\section{References}

1. Herr K, Coyne PJ, Ely E, McCaffery M, Manworren R, Merkel S. Pain assessment in the patient unable to self-report: Position statement with clinical practice recommendations. Pain Manag Nurs. 2011; 12: 230-250.

2. AGS Panel on Persistent Pain in Older Persons. The management of persistent pain in older persons. J Am Geriat Soc. 2002; 50: S205-S224.

3. Lichtner V, Dowding D, Esterhuizen P, Closs SJ, Long AF, Corbett A, et al. Pain assessment for people with dementia: A systematic review of systematic reviews of pain assessment tools. BMC Geriatr. 2010; 14: 138.

4. Richaud L. Prise en charge antalgique de la personne âgée en structure médicalisée: État des lieux en France. Marseille: Aix Marseille University; 2019. Available from: https://dumas.ccsd.cnrs.fr/dumas-02388956.

5. Vieillard $M$, Rat $P$, Jouve $E$, Lacroix $O$, Rossi $P$, Bonin-Guillaume S. Appropriation de l'échelle ALGOPLUS par les soignants. Rev Geriatr. 2016; 41: 467-476.

6. Hadjistavropoulos T, Herr K, Turk DC, Fine PG, Dworkin RH, Helme R, et al. An interdisciplinary expert consensus statement on assessment of pain in older persons. Clin J Pain. 2007; 23: S1S43.

7. Hadjistavropoulos T, Voyer P, Sharpe D, Verreault R, Aubin M. Assessing pain in dementia patients with comorbid delirium and depression. Pain Manag Nurs. 2008; 9: 48-54.

8. Hung M, Bounsanga J, Liu F, Voss MW. Profiling arthritis pain with a decision tree. Pain Pract. 2018; 18: 568-579.

9. Ward CW. A decision tree model for postoperative pain management. Medsurg Nurs. 2015; 24: 77-82.

10. Hu YJ, Ku TH, Jan RH, Wang KC, Tseng YC, Yang SF. Decision tree-based learning to predict patient controlled analgesic consumption and readjustment. BMC Med Inform Decis Mak. 2012; 12: 131.

11. Wright R, Malec M, Shega JW, Rodriguez E, Kulas J, Morrow L, et al. Deconstructing chronic low back pain in the older adult-step by step evidence and expert-based recommendations for evaluation and treatment: Part XI: Dementia. Pain Med. 2016; 17: 1993-2002. 
12. Rat $P$, Jouve E, Pickering $G$, Donnarel D, Nguyen L, Michel M, et al. Validation of an acute painbehavior scale for older persons with inability to communicate verbally: Algoplus ${ }^{\circledR}$. Eur J Pain. 2011; 15: 198-204.

13. Bonin-Guillaume S, Jouve E, Lauretta R, Nalin C, Truillet R, Capriz F, et al. Algoplus performance to detect pain in depressed and/or demented old patients. Eur J Pain. 2016; 20: 1185-1193.

14. Monacelli F, Signori A, Roffredo L, Pace K, Nencioni A, Pickering G, et al. Algoplus ${ }^{\circledR}$ scale in older patients with dementia: A reliable real-world pain assessment tool. J Alzheimers Dis. 2017; 56: 519-527.

15. Pickering G, Monacelli F, Pérez-Castejón Garrote JM, Guarda H, Batalha L, Gibson S, et al. Reliability study in five languages of the translation of the pain observational scale Algoplus. Pain Med. 2018; 19: 252-261.

16. Rat $\mathrm{P}$, Jouve $\mathrm{E}$, Bonin-Guillaume $\mathrm{S}$, Doloplus $\mathrm{C}$. A cut-off score of Algoplus to assess pain in elderly patients and define the need to prescribe analgesia. Eur J Pain. 2011; 1: 246.

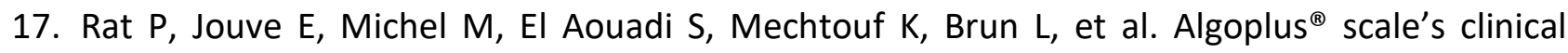
appropriateness: Does it require specific training before use? 13th World Pain Congress IASP; 2010 August 29; Montréal.

18. Martin E, Doloplus Collective team, Pereira B, Pickering G. Concordance of pain detection using the Doloplus and Algoplus behavioral scales. J Am Geriatr Soc. 2016; 64: e100-e102.

19. Rat $P$, Bonin-Guillaume S, Pickering G, Leglise MS, Doloplus C. Algorithm for pain assessment in elderly patients. Douleurs. 2014; 15: 52-56.

20. American Geriatrics Society Panel on Pharmacological Management of Persistent Pain in Older Persons. Pharmacological management of persistent pain in older persons. J Am Geriatr Soc. 2009; 57: 1331-1346.

21. Évaluation et prise en charge th rapeutique de la douleur chez les personnes âgées ayant des troubles de la communication verbale [Internet]. Denis La Plaine Cedex: Haute Autorité De Santé; 2000. Available from: https://www.has-sante.fr/icms/c 272123/fr/evaluation-et-priseen-charge-therapeutique-de-la-douleur-chez-les-personnes-agees-ayant-des-troubles-de-lacommunication-verbale.

22. Payen JF, Bru O, Bosson JL, Lagrasta A, Novel E, Deschaux I, et al. Assessing pain in critically ill sedated patients by using a behavioral pain scale. Crit Care Med. 2001; 29: 2258-2263.

23. Kotfis K, Strzelbicka M, Zegan-Barańska M, Safranow K, Brykczyński M, Żukowski M, et al. Validation of the behavioral pain scale to assess pain intensity in adult, intubated postcardiac surgery patients: A cohort observational study-POL-BPS. Medicine. 2018; 97: e12443.

24. Caltagirone C, Spoletini I, Gianni W, Spalletta G. Inadequate pain relief and consequences in oncological elderly. Surg Oncol. 2010; 19: 178-183. 


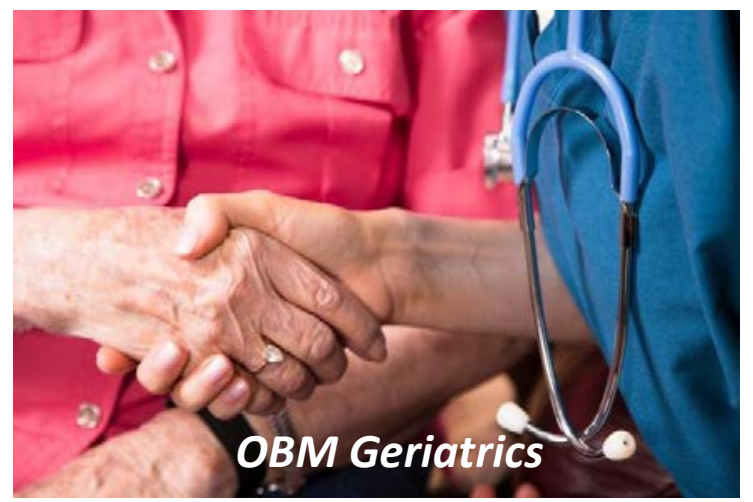

Enjoy OBM Geriatrics by:

1. Submitting a manuscript

2. Joining in volunteer reviewer bank

3. Joining Editorial Board

4. Guest editing a special issue

For more details, please visit: http://www.lidsen.com/journals/geriatrics 